\title{
Studying the Effect of Magnetic Strength and Electrical Conductivity on the Velocity Profile of an MHD Flow in a Rectangular Duct
}

\author{
OMAR YOUSEF AL-THEHABEY \\ Department of Aerospace Engineering, University of Cincinnati, Cincinnati, OH United States
}

\begin{abstract}
This document presents the results of an investigation of the impact of variations of electrical conductivity and magnetic field strength on the velocity profile inside a rectangular duct, assuming equilibrium flow. It also presents the physics and the complete theory associated with the MHD, then derive the equations to be utilized for computational analysis of the magnetic field and the velocity profiles. The computational work in this document is based on the assumption that the working fluid is air and it is fully developed in the duct. The finite difference method is used to perform the analysis for 2 distinct cases: the first case is when the magnetic field strength changes, while holding the electrical conductivity constant. The second case is when the electrical conductivity is changing while holding the magnetic field strength constant.It has been found that increasing the magnetic field strength has resulted in decreasing the axial velocity profile, which demonstrated that the magnetic field was pulling the flow to its direction, reducing the flow momentum in the axial direction. It has also been found that the electrical conductivity reduced the velocity profile in the axial direction when that conductivity was increasing, demonstrating that the electric field was also pulling the flow in its direction, reducing the flow momentum in the axial direction.
\end{abstract}

Keywords: MHD, Magnetohydrodynamics, Flow in Rectangular Duct, Magnetic Field, Electrical Conductivity, MHD Flow, MHD Flow Velocity Profile

DOI: $10.7176 / \mathrm{JETP} / 10-4-03$

Publication date:August $31^{\text {st }} 2020$

\section{Introduction}

MHD stands for magneto-hydrodynamic, also called magneto-gas-dynamics, magneto-aerodynamics or magnetoplasma aerodynamics. It is used to propel vehicles in the air, water, or space, using electric and magnetic fields only, by accelerating an electrically conductive propellant, liquid or gas, then the fluid is directed to the rear, which results in accelerating the vehicle forward, as a reaction. MHD was first tested in the field of marine propulsion in the early 1960s. However, due to the low electrical conductivity of seawater, the application of MHD propulsion to marine vehicles was inefficient and therefore impractical as increasing current density was limited by Joule heating and water electrolysis near the electrodes. In addition, increasing the magnetic strength was associated with an increase in cost, size, and weight of electromagnets. The application of MHD in air-breathing propulsion is still limited to theoretical concepts, whereas in space propulsion, MHD has been used to produce high thrust and high specific impulse, and the propellant would last much longer than chemical rockets.

In air-breathing engines, such as conventional gas-turbine engines, electrical power is produced using mechanically rotating generators. In ramjet and scramjet engines, where there are no rotating parts, electrical power is produced via another mechanism: the working fluid, which is air, is ionized in order to become electrically conductive. At high altitudes, this ionization is achieved using special techniques, such as high-voltage electric arc discharge, radio frequency electromagnetic glow discharge, laser, e-beam, and other techniques. The goal of MHD application here is to achieve higher Mach numbers. The reduction of airflow velocity upstream of scramjet inlet is made by the use of an MHD generator, then followed by an MHD accelerator downstream in the exhaust nozzle. An example of this design is the Russian AJAX and the US hypersonic vehicle electric power system (HVEPS) projects.

The concept of introducing strong applied electric and magnetic fields into flow serves two things: it can reduce the heat damage by providing sort of magneto-hydrodynamic parachute, and it can generate an electric power that can be used to increase the vehicle thrust as well as performing some electrical functionality. The principle components of an MHD generator are an electrode-lined channel inside a superconducting magnet.

\section{Research Contributions}

Investigating the impact of Mach number, electrical conductivity, and magnetic field strength on the performance of MHD devices.

\section{Previous Studies}

Zhang et al (2019), used numerical computations, an edge-based finite element compressible turbulent solver for hypersonic flows, to obtain results for an MHD converging channel accelerator: a 2-D inviscid flow in a scramjet, 
and a 3-D viscous flow in the same scramjet. The numerical results are compared against published numerical ones and show good agreement.

Fomichev and Yadrenkin (2017) in their experimental work, studied the interaction of an electric discharge with a hypersonic flow and a magnetic field. They have shown that the work of the volumetric electromagnetic force leads to the deceleration of the hypersonic air flow, to the fixation of the ionization region in the flow, to the change of pressure in the interaction zone and to the appearance of a bow shock wave in front of the interaction zone. They have also shown that at strong MHD-interaction, the shape of the model slightly influences the final result of the change in the flow pattern. However, their studies were carried out on an experimental MHD test rig based on a shock tube.

Lee, Huerta, and Zha (2010), presented a 3-D hypersonic laminar flows of air around blunt bodies with applied magnetic fields of different strengths, using a shock to heat air. Their study is totally computational using an upwind scheme and their results did not show any correlation between magnetic strength and changes in air properties (temperature and pressure).

Balasubramanian et al (2017), carried out a numerical investigation to demonstrate an MHD-based active flow control to mitigate the laminar flow separation over a flat plate due to shock-wave boundary layer interaction. Their computational results showed that skin friction and drag increased drastically compared with a non-MHD case.

Pengyu et al (2016), demonstrated an MHD power generation with supersonic non-equilibrium plasma. In an MHD channel of $16 \mathrm{~mm} \times 10 \mathrm{~mm} \times 20 \mathrm{~mm}$, MHD open voltage of $10 \mathrm{~V}$ was realized in the magnetic field of 1.25 $\mathrm{T}$, and a power of $0.12 \mathrm{~mW}$ was steadily and continuously extracted in the magnetic field of $1 \mathrm{~T}$. The reasons for limited power generation were proposed as: low conductivity of RF discharge; large touch resistance between MHD electrode and plasma; strong current eddies due to flow boundary layer. In addition, the cathode voltage fall is too low to have obvious effects on MHD power generation.

Balasubramanian et al (2016), had computationally studied the MHD power generation for controlling and augmenting the hypersonic flow, as well as for thermal management schemes, using a CERANS-MHD code. The concept was conceived to use the existing adverse hypersonic environment around the vehicle toward its advantage. The AJAX concept made extensive utilization of MHD-based flow control techniques, where the fluid that was pre-ionized and made electrically conducting, was easily manipulated by an externally applied magnetic field. Hence, the hypersonic flow field could be easily modified for convenience, even at off-design flight conditions, by application of a suitable magnetic field.

Chutial and Deka (2014), studied a steady MHD flow for an incompressible, viscous, electrically conducting fluid in a rectangular duct. Their computations have been carried out for several values of the Hartmaan number $(\mathrm{H})$, magnetic Reynolds number $(\mathrm{Rm})$, and the aspect ratio $\mathrm{A}=\mathrm{b} / \mathrm{a}$, for velocity and induced magnetic field. Their results indicate that aspect ratio A may play a significant role on the solutions of the duct flow, besides the other parameters $\mathrm{H}$ and $\mathrm{Rm}$ associate with external magnetic field strength.

Shah, Hussain, and Sagheer (2016), presented a numerical analysis of MHD flow of Maxwell fluid with thermal radiation and Joule heating by considering the recently developed Cattaneo-Christov heat flux model which explains the time relaxation characteristics for the heat flux. Their results showed stronger magnetic parameter $\mathrm{M}$ increased temperature and decreased velocity: temperature profile increases while increasing the radiation parameter and the similar effect of Eckert number is observed on the temperature field, and velocity in the horizontal direction decreases while temperature increases.

Lindsey (2006), investigated three distinct applications: stand-alone MGD power generation performed upstream of the combustor, stand-alone combustor-based MGD power generation, and a full MGD energy bypass system. This study encountered performance penalties in terms of a drag increase, as well as dissipation induced heating, reduced most of the overall inlet performance measures with respect to the no-MGD baseline. Comparing combustor-based MGD power generation to its isolator-based counterpart revealed that the combustor-based generators performed moderately better, in spite of localized pressure increases in the vicinity of the electrodes which indicated the presence of small shock waves.

Shneider et al (2004), focused on the on-ramp MHD generator that would bring the shocks back on lip at Mach numbers greater than the design value. Compared with the earlier work, here this study emphasized and used advantages of a very short $(15-30-\mathrm{cm})$ MHD interaction region. The study found the performance of the short MHD region is enhanced by operating upstream, at the first compression ramp, and by using the strongest magnetic field near the coil centerline.

\section{Physics of Magneto-Hydrodynamics}

This is concerned the dynamics of magnetic fields in electrically conducting fluid, such as air. Physicist Hannes Alfven (1908-1995) presented some fundamental work and discoveries in MHD with applications in different parts of plasma physics. The basic theory of MHD is that magnetic fields, supported by conductive fluids, produce forces that in turn act on the fluid, and consequently altering the topology and strength of the magnetic fields. 
In hypersonic flight, shocks generated change the thermodynamic properties of the air, which is heated, and high temperature changes the specific heats of the air, treated as an ideal gas (chemically frozen) or real gas. These changes in air properties are associated with rotational excitation, vibrational excitation, molecular dissociation, molecular ionization, and other effects. As a result of the ionization of air molecules, they become electrically conducting producing an electric current that has two main benefits: reducing the heat damage by providing sort of magneto-hydrodynamic parachute, and generate an electric power that can be used to thrust the vehicle, as well as providing some other electrical functionality. Figure 1, below, shows the sequence how a hypersonic flow can lead to generating magnetism.

Describing the flow fields must take into accounts the shock discontinuities before simulating the hypersonic flow with the MHD effects. That is, to incorporate the magnetic effects and the thermodynamics of high temperature flow in one algorithm. Therefore, the inviscid heat flux will be split into two components: convective flux and pressure flux. When considering air as an ideal gas (chemically frozen), high temperature effects will be neglected. However, by considering air as a real gas, behind the hypersonic bow shock, the high temperature causes molecular dissociation, chemical reactions, ionization, and other effects that change the equation of state and the energy equation of the air. These effects require the accounting for the reaction rate equation for each chemical species, the rotational and vibrational energy equations, and the presence of more than one temperature.

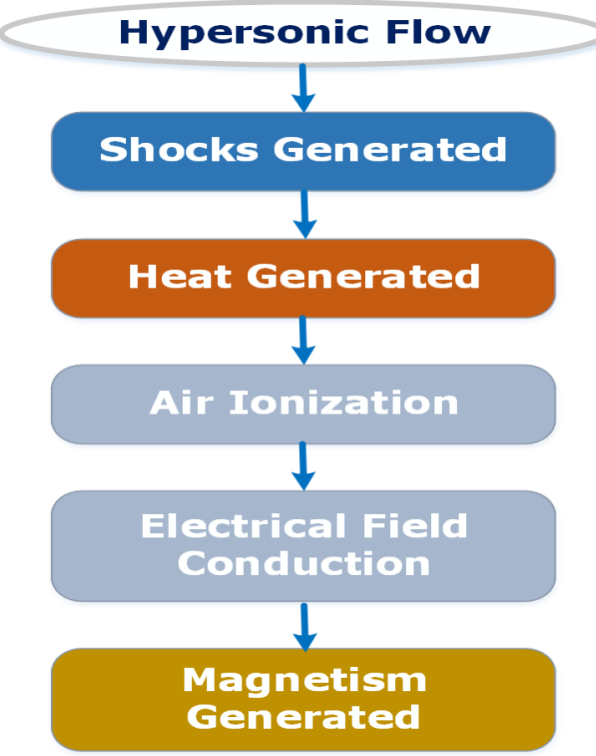

Figure 1: Sequence how a hypersonic flow can lead to generating magnetism.

It would be noteworthy to list the differences, shown in Table 1 below, between ideal and real air, so that for each case, appropriate steps will be taken.

Table 1: Ideal gas vs. real gas.

\begin{tabular}{|l|l|}
\hline Ideal Gas (Hypothetical) & Real Gas \\
\hline Indefinite volume and high pressure. & Definite volume and lower pressure. \\
\hline Particles have elastic collisions. & Particles have inelastic collisions. \\
\hline No intermolecular attraction forces & Intermolecular attraction forces exist \\
\hline Obeys P V = n R T & Does not obey P V =n R T \\
\hline
\end{tabular}

\section{The MHD Model}

For describing an equilibrium model for the MHD flow, it is assumed that there is enough time for the gas to reach local thermodynamic equilibrium. Then the pressure and temperature can be determined in terms of the specific heat ratio, $\gamma$. For the viscosity calculation, the Sutherland's law as the air plasma obeys Ohm's law. Another assumption is that the displacement current is small enough to be neglected, and the electric conductivity, $\mathrm{k}$, in the model is constant. The main quantities used in this model include the magnetic field $\mathbf{B}$, the electrical field $\mathbf{E}$, the current density $\mathbf{J}$, the fluid density $\rho$, the fluid dynamic viscosity $\mu$, the pressure $\mathrm{p}$, the velocity $\mathbf{V}$, the energy per unit mass e, the heat flux vector $\mathrm{q}$, and the viscosity tensor $\tau$.

Statement of the Problem:

The problem consists of a rectangular duct of 1 unit width and 1 unit height, but an infinite length. All duct sides are electrically insulated. The flow driving force is a constant applied pressure gradient $(\partial \mathrm{p} / \mathbf{\partial z})$. The flow is electrically conducting, incompressible and viscous air, under the action of an external uniform transverse magnetic field transverse to the direction of the flow. It is required to find the effect of different strengths of 
magnetic field, $\mathbf{B}_{\mathbf{0}}$, and electrical conductivity, $\boldsymbol{\sigma}$, on the velocity profile.

\section{Assumptions:}

1. Working fluid is air, steady, fully developed and under standard sea-level conditions.

2. Flow is incompressible and viscous.

3. Magnetic field is uniform from the inlet of the duct to the end of the duct.

4. Velocity profile stays constant throughout the duct in the axial direction, the duct is smooth and no friction.

5. The pressure gradient is dependent on $\mathrm{z}, \partial \mathrm{p} / \partial \mathrm{x}$ and $\partial \mathrm{p} / \partial \mathrm{y}$ are negligible.

6. The magnetic field is constant, $\mathbf{B}_{\mathbf{0}}$, in the $\mathrm{x}$, but changing the z-direction.

\section{Governing Equations}

The governing equations for the magneto-hydrodynamic flow are a combination of the Navier-Stokes and the Maxwell's equations. As a result of this combination, we get

$$
\begin{gathered}
\boldsymbol{\nabla} . \mathbf{B}=0 \\
\boldsymbol{\nabla} . \mathbf{V}=0 \\
\mathbf{J}=\boldsymbol{\sigma}(\mathbf{E}+\mathbf{V} \times \mathbf{B}) \\
\mathbf{V} \times \mathbf{B}=\mu_{e} \mathbf{J} \\
\frac{\partial \boldsymbol{V}}{\partial t}+(\boldsymbol{V} . \nabla) V=-\frac{1}{\rho}\left[\nabla \mathrm{p}-\mathbf{J} \times \mathbf{B}-\mu \nabla^{2} \boldsymbol{V}\right] \\
\frac{\partial \boldsymbol{B}}{\partial t}=\nabla \times(\boldsymbol{V} \times \boldsymbol{B})+\lambda \nabla^{2} \boldsymbol{B}
\end{gathered}
$$

Here, the bold character indicates the stated quantity is in vector form.

\section{Initial Conditions:}

The velocity at the side walls is zero. Also, the magnetic field at the sides is zero.

\section{Numerical Methods Application}

We will utilize the finite difference to discretize the MHD equations, above, in the same manner used by Chutia1 and Deka (2014), but they used the central difference method. If s denotes the length and the width of the side of the rectangle, then $\mathrm{A}$ as the aspect ratio, where $\mathrm{A}=\mathrm{s} / \mathrm{s}=1$. Also, $\mathrm{Rm}$ denote the Reynolds number, where $\mathrm{Rm}=$ $\mu V_{0} s$, then we get the following equations for the first and the second derivatives of the velocity vector:

$\frac{\partial V}{\partial x}=\frac{V_{i+1, j}-V_{i-1, j}}{2 \Delta x}+O\left(\Delta x^{2}\right)$

$\frac{\partial V}{\partial y}=\frac{V_{i, j+1}-V_{i, j-1}}{2 \Delta y}+O\left(\Delta y^{2}\right)$

$\frac{\partial^{2} V}{\partial x^{2}}=\frac{V_{i+1, j}-2 V_{i, j}+V_{i-1, j}}{\Delta x^{2}}+O\left(\Delta x^{2}\right)$

$\frac{\partial^{2} V}{\partial y^{2}}=\frac{V_{i, j+1}-2 V_{i, j}+V_{i, j-1}}{\Delta y^{2}}+O\left(\Delta y^{2}\right)$

Similarly, we obtain the first and the second derivatives of the magnetic field vector:

$\frac{\partial B}{\partial x}=\frac{B_{i+1, j}-B_{i-1, j}}{2 \Delta x}+O\left(\Delta x^{2}\right)$

$\frac{\partial B}{\partial y}=\frac{B_{i, j+1}-B_{i, j-1}}{2 \Delta y}+O\left(\Delta y^{2}\right)$

$\frac{\partial^{2} B}{\partial x^{2}}=\frac{B_{i+1, j}-2 B_{i, j}+B_{i-1, j}}{(\Delta x)^{2}}+O(\Delta x)^{2}$

$\frac{\partial^{2} B}{\partial y^{2}}=\frac{B_{i, j-1}-2 B_{i, j}+B_{i, j-1}}{(\Delta y)^{2}}+O(\Delta y)^{2}$

Here, $O$ denotes the error, which is negligible. Now, we can use the discretization in equations (16) and (17) of Chutial and Deka (2014).

$\left[\frac{V_{i+1, j}-2 V_{i, j}+V_{i-1, j}}{(\Delta x)^{2}}+\frac{1}{A^{2}}\left(\frac{V_{i, j+1}-2 V_{i, j}+V_{i, j-1}}{(\Delta y)^{2}}\right)\right]+\frac{H^{2}}{R m}\left(\frac{B_{i+1, j}-B_{i-1, j}}{2 \Delta x}\right)+1=0$
$\left[\frac{B_{i+1, j}-2 B_{i, j}+B_{i-1, j}}{(\Delta x)^{2}}+\frac{1}{A^{2}}\left(\frac{B_{i, j+1}-2 B_{i, j}+B_{i, j-1}}{(\Delta y)^{2}}\right)\right]+R m\left(\frac{V_{i+1, j}-V_{i-1, j}}{2 \Delta x}\right)=0$

Here, $H$ is the Hartmann number $\left(H=B_{0} L \sqrt{\frac{\sigma}{\rho v}}\right)$ and not $\left(H=B_{0} L\left(\frac{\sigma}{\rho v}\right)^{2}\right)$, as used incorrectly in Chutial and

Deka (2014), $\mathrm{Rm}$ is the magnetic Reynolds number $\left(\mathrm{Rm}=\mu \sigma V_{0} L\right.$, where $\mathrm{L}$ is the characteristic length, in this case $\mathrm{L}=\mathrm{s}$.

Now, using equations (15) and (16), we can express the velocity and the magnetic field vectors in a discretized form and apply the finite difference method using the initial and the boundary conditions stated above. First, let's define the following constants, $\mathrm{C}_{1}$ to $\mathrm{C}_{5}$.

$C_{1}=\frac{A^{2}(\Delta y)^{2}}{2\left[A^{2}(\Delta y)^{2}+(\Delta x)^{2}\right]}, \quad C_{2}=\frac{(\Delta x)^{2}}{2\left[A^{2}(\Delta y)^{2}+(\Delta x)^{2}\right]}$,

$C_{3}=\frac{H^{2} A^{2} \Delta x(\Delta y)^{2}}{4 R m\left[A^{2}(\Delta y)^{2}+(\Delta x)^{2}\right]}, \quad C_{4}=\frac{A^{2}(\Delta x)^{2}(\Delta y)^{2}}{2\left[A^{2}(\Delta y)^{2}+(\Delta x)^{2}\right]}, \quad C_{5}=\frac{R m A^{2} \Delta x(\Delta y)^{2}}{4\left[A^{2}(\Delta y)^{2}+(\Delta x)^{2}\right]}$

Now, $V_{i, j}$ and $B_{i, j}$ can be expressed in terms of these constants:

$V_{i, j}=C_{1}\left[V_{i+1, j}+V_{i-1, j}\right]+C_{2}\left[V_{i, j+1}+V_{i, j-1}\right]+C_{3}\left[B_{i+1, j}-B_{i-1, j}\right]+C_{4}$ 
$B_{i, j}=C_{1}\left[B_{i+1, j}+B_{i-1, j}\right]+C_{2}\left[B_{i, j+1}+B_{i, j-1}\right]+C_{5}\left[V_{i+1, j}-V_{i-1, j}\right]$

Now, the system of equations (17) and (18) can be solved using finite difference method.

\section{Discussion of Results}

The application was run for two cases: variable magnetic field strength () and variable electrical conductivity. Using the finite difference method, at iteration 100 , the solution was reached and the velocity profile was obtained. Figures 2 through 5, below, show the results for case 1 , at $\mathrm{B}_{0}$ of $0.001,0.002,0.003$, and $0.005 \mathrm{~T}$ at $10,000 \mathrm{~N} . \mathrm{s} / \mathrm{C} . \mathrm{m}$ electrical conductivity. In this run, the axial velocity profile decreased when magnetic field strength increased. This demonstrates that the magnetic field is pulling the flow towards its direction away from the z-direction, and therefore, due to this magnetic force, the velocity decreased. The listed tabulated values of average and maximum velocity on the left side of the figures shows the decrease when moving from one magnetic strength to the higher strength.

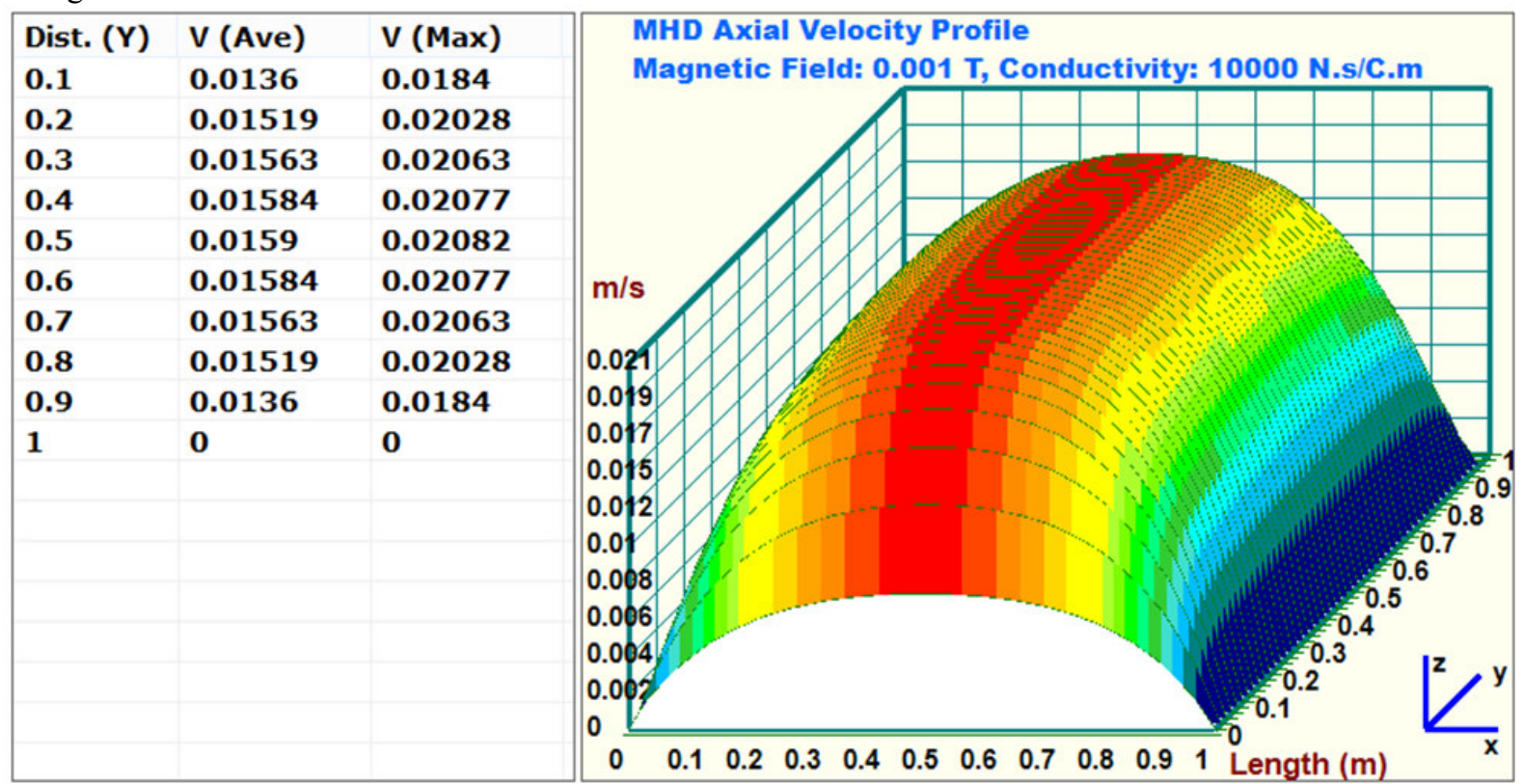

Figure 2: Axial velocity profile at magnetic field $\mathrm{B}_{0}=0.001 \mathrm{~T}$ and $\mathrm{k}=10,000 \mathrm{~N} . \mathrm{s} / \mathrm{C} . \mathrm{m}$.

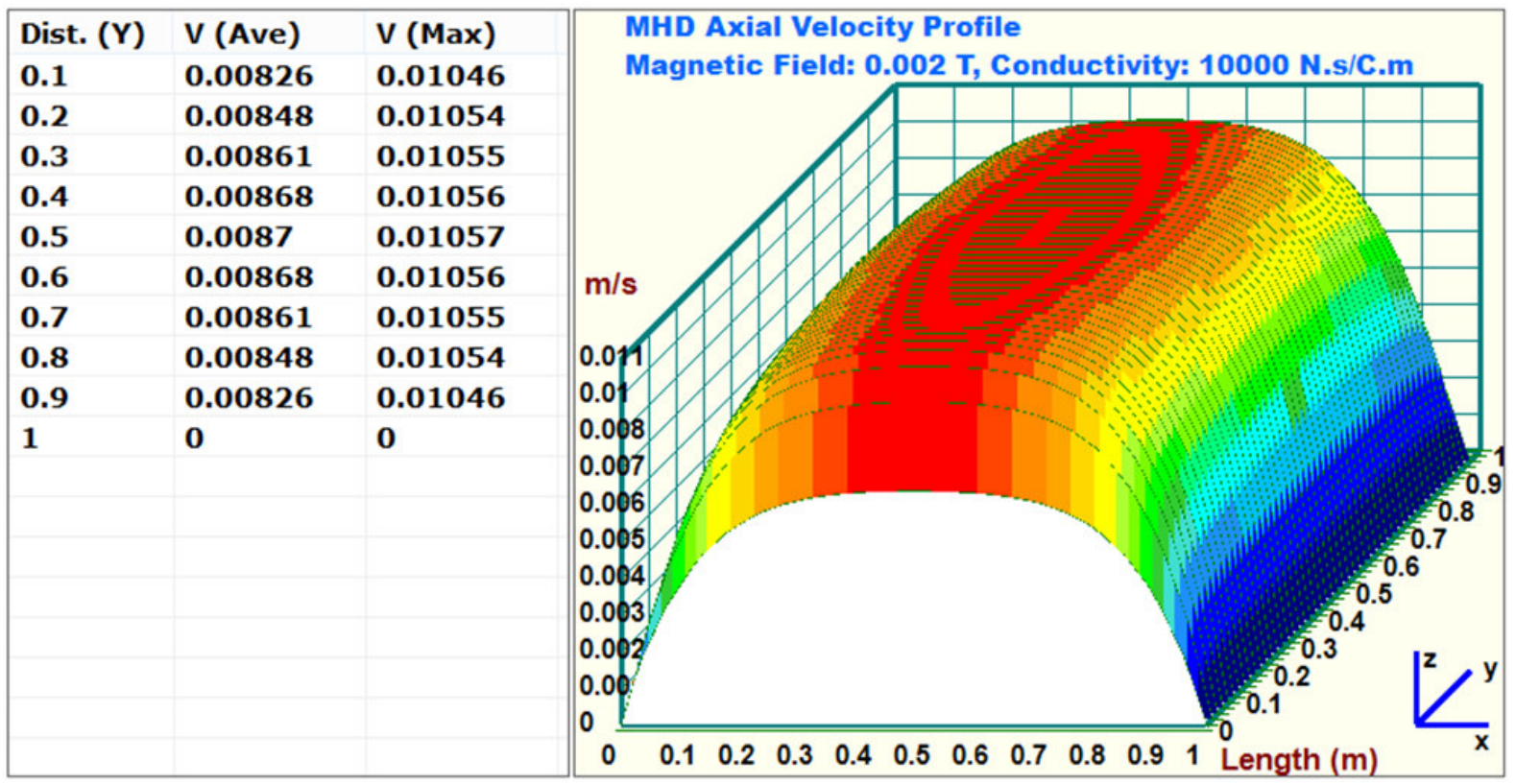

Figure 3: Axial velocity profile at magnetic field $\mathrm{B}_{0}=0.002 \mathrm{~T}$ and $\mathrm{k}=10,000 \mathrm{~N} . \mathrm{s} / \mathrm{C} . \mathrm{m}$. 


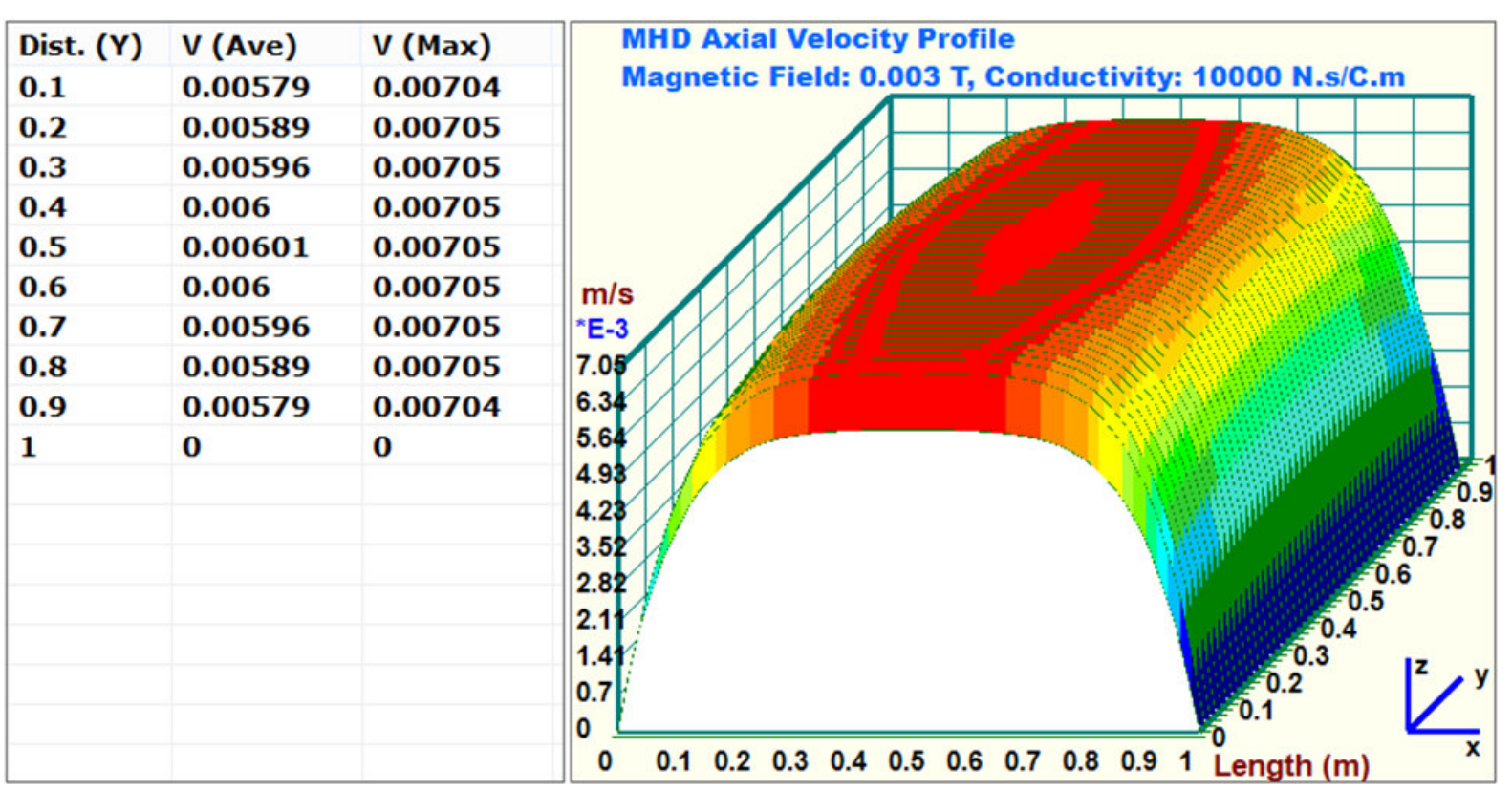

Figure 4: Axial velocity profile at magnetic field $\mathrm{B}_{\mathbf{0}}=0.003 \mathrm{~T}$ and $\mathrm{k}=10,000$ N.s/C.m.

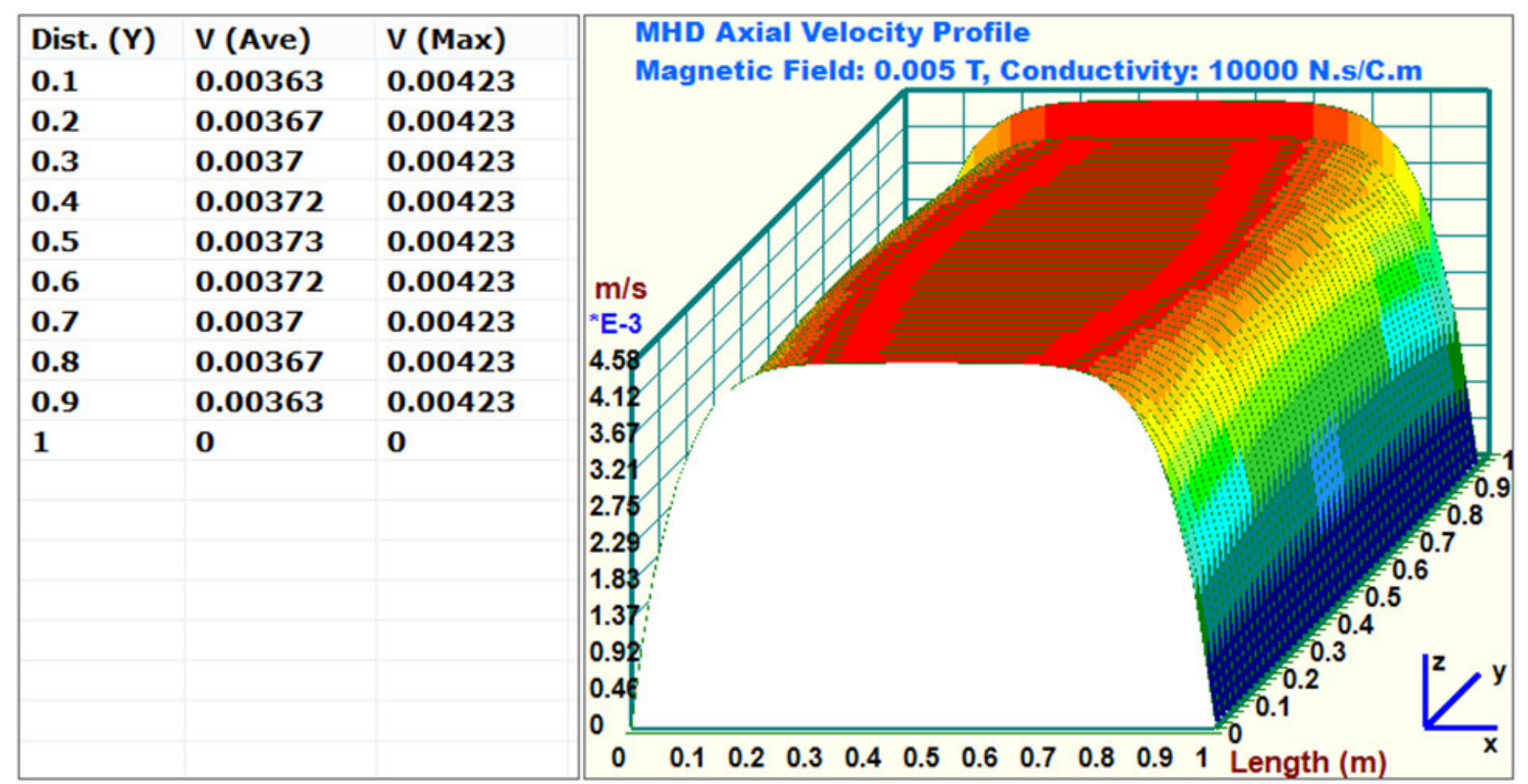

Figure 5: Axial velocity profile at magnetic field $\mathrm{B}_{0}=0.005 \mathrm{~T}$ and $\mathrm{k}=10,000$ N.s/C.m.

Figures 6 through 9 below show the results for case 2, at a magnetic field strength of $0.003 \mathrm{~T}$ for different electrical conductivity: $15,000,20,000,25,000$, and 30,000. In this run, it was noticed that increasing electrical conductivity contributed to decreasing velocity in the axial direction. That indicates another force, the magnetic force, was pulling the velocity profile in its direction, resulting a reduction in the axial direction. The listed tabulated values of average and maximum velocity on the left side of the figures shows the decrease when moving from one electrical conductivity to the higher value. 


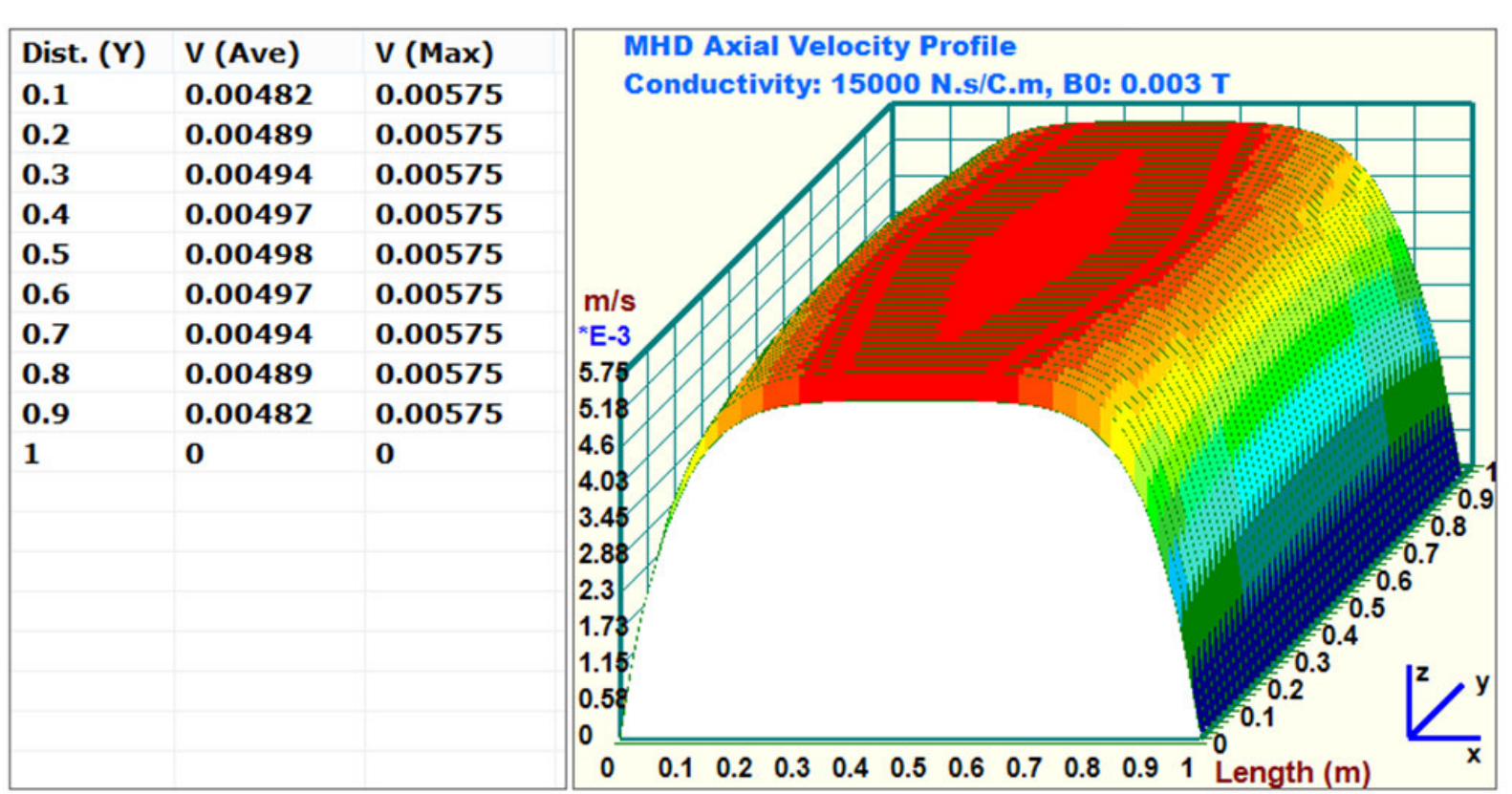

Figure 6: Axial velocity profile at magnetic field $\mathrm{B}_{\mathbf{0}}=0.003 \mathrm{~T}$ and $\mathrm{k}=15,000$ N.s/C.m.

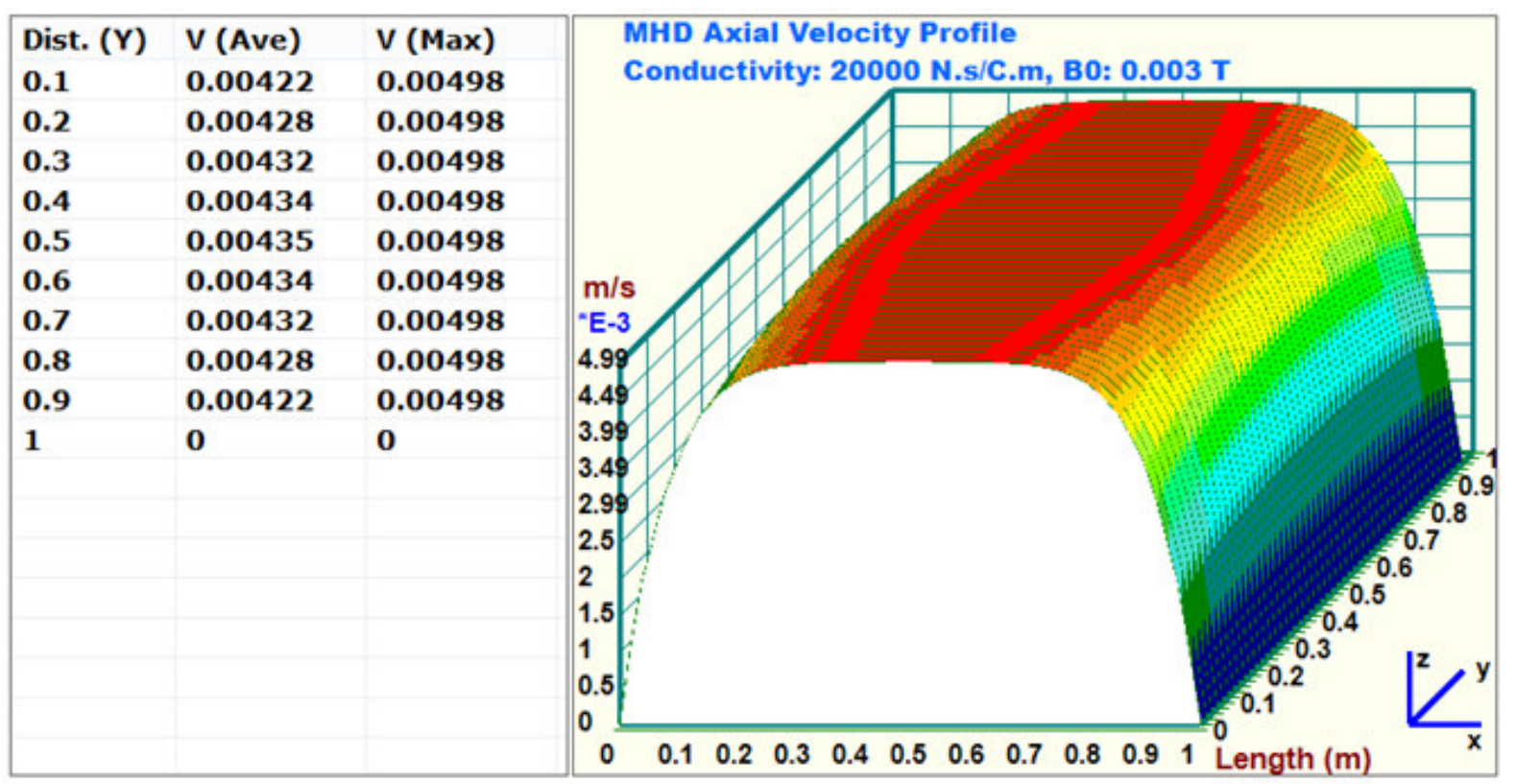

Figure 7: Axial velocity profile at magnetic field $\mathrm{B}_{0}=0.003 \mathrm{~T}$ and $\mathrm{k}=20,000$ N.s/C.m. 


\begin{tabular}{|l|l|l|}
\hline Dist. (Y) & V (Ave) & V (Max) \\
0.1 & 0.00381 & 0.00446 \\
0.2 & 0.00386 & 0.00446 \\
0.3 & 0.00389 & 0.00446 \\
0.4 & 0.00391 & 0.00446 \\
0.5 & 0.00392 & 0.00446 \\
0.6 & 0.00391 & 0.00446 \\
0.7 & 0.00389 & 0.00446 \\
0.8 & 0.00386 & 0.00446 \\
0.9 & 0.00381 & 0.00446 \\
1 & 0 & 0 \\
\hline
\end{tabular}

10

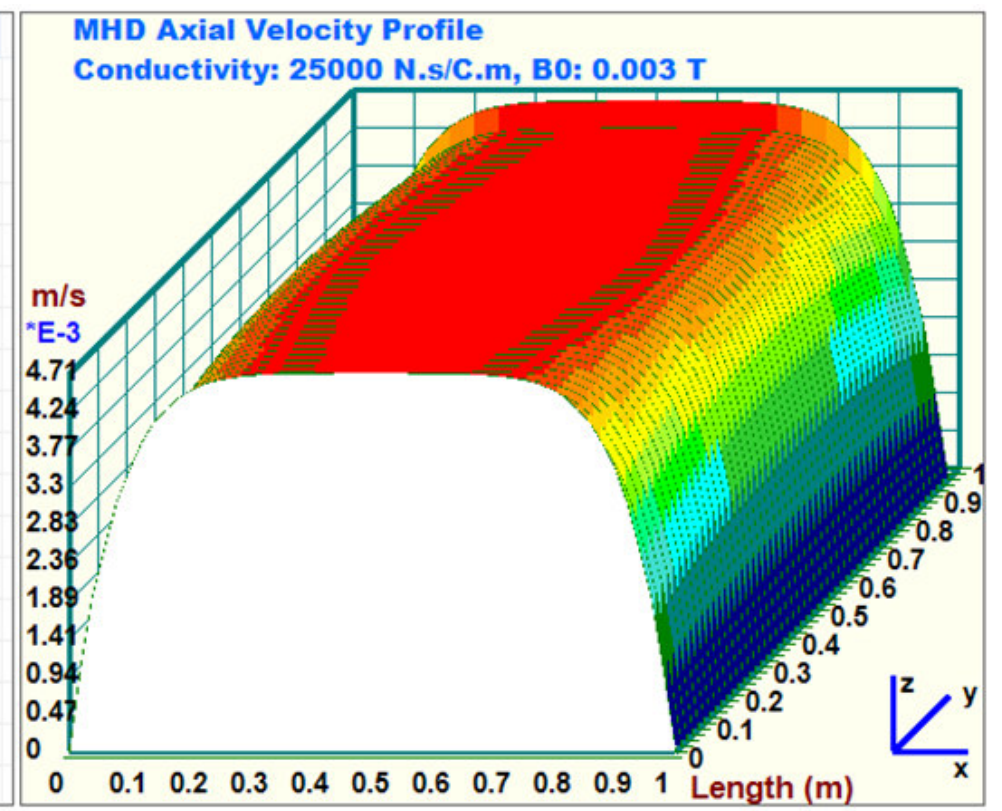

Figure 8: Axial velocity profile at magnetic field $\mathrm{B}_{0}=0.003 \mathrm{~T}$ and $\mathrm{k}=25,000$ N.s/C.m.

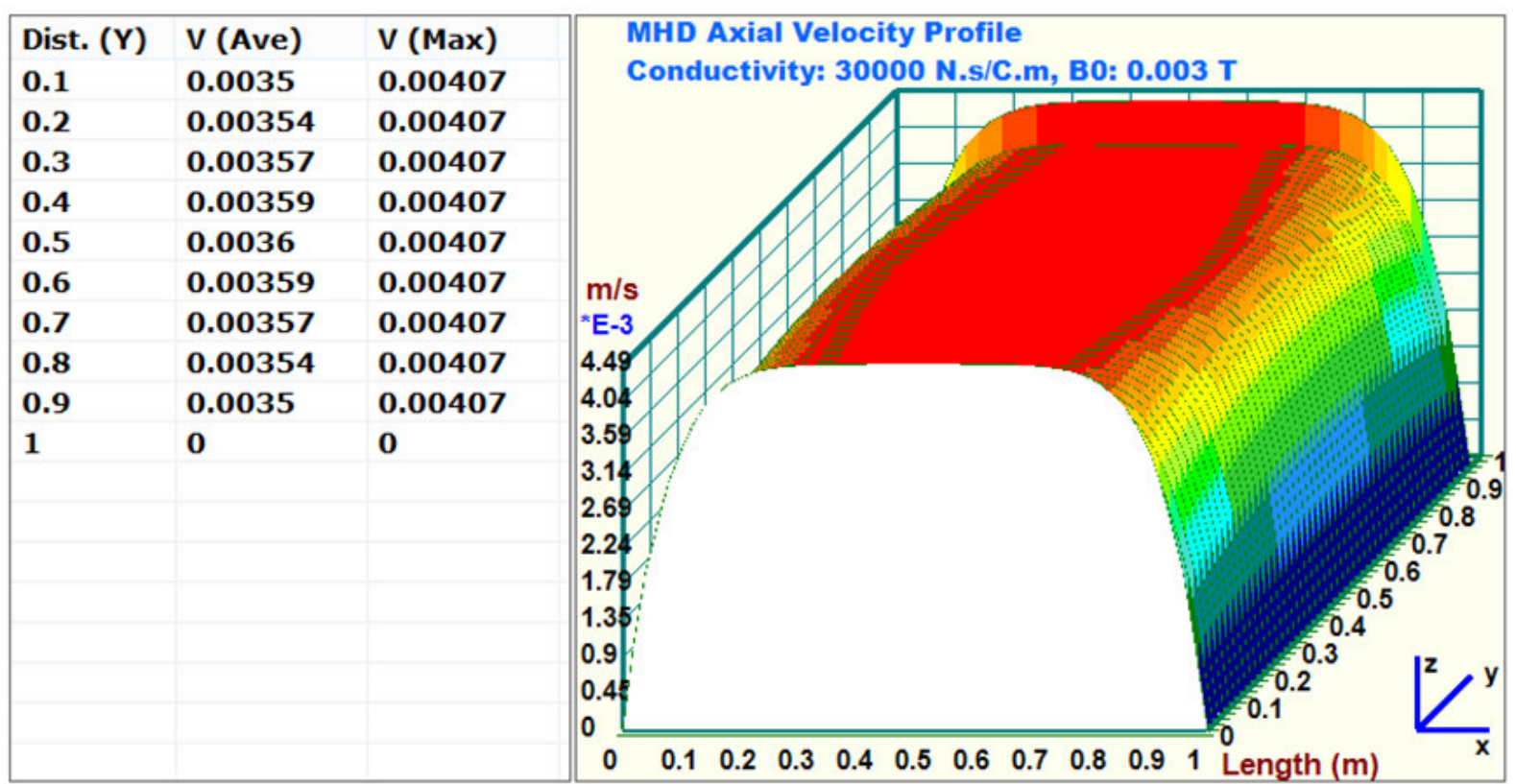

Figure 9: Axial velocity profile at magnetic field $\mathrm{B}_{0}=0.003 \mathrm{~T}$ and $\mathrm{k}=30,000$ N.s/C.m.

\section{Results Validation}

We compared our results with the results in Chutia1 and Deka (2014). We used different set of constant than those presented in that article. Now, using the new constants, we were able to produce the charts presented in the figures 3,8 , and 9 after using the values of electrical conductivity and magnetic strength that would result in equivalent values of Hartmann and magnetic Reynolds numbers used in that article. For example, the following chart corresponds to Figure 10, with Rem $=1.0$ and $\mathrm{H}=150$ after 15 iterations. Our results is very close to Chutia1 and Deka (2014). 


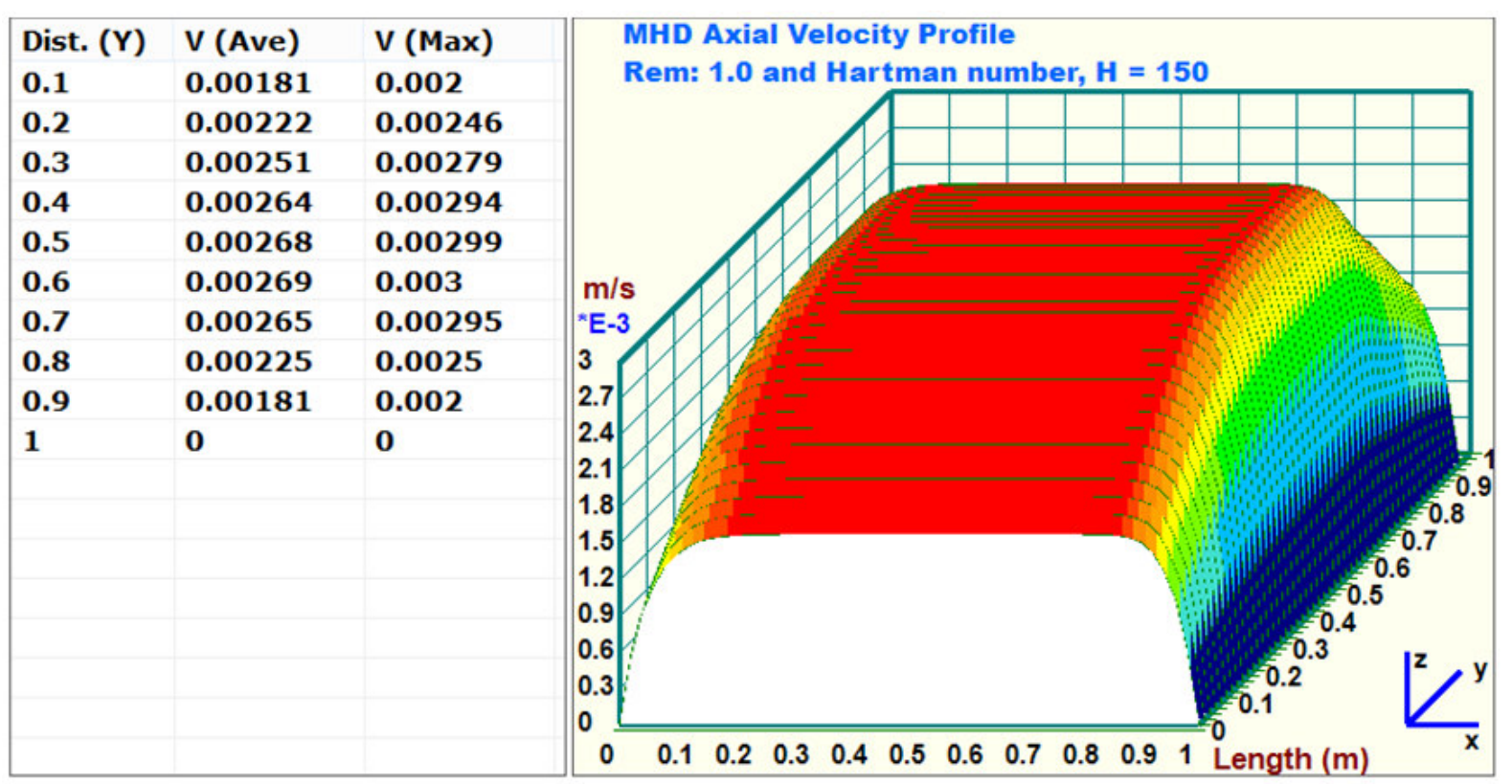

Figure 10: Comparing results with Figure 10 in Chutia1 and Deka (2014) article.

\section{Conclusion}

Based on the computation performed, it is shown that increasing the magnetic field strength decreased the axial velocity by reducing the flow momentum in the axial direction. Similar effect was found for increasing the electrical conductivity, it reduced the axial velocity of the flow. The MHD impact can be further investigated in regards to temperature and pressure.

\section{References}

Anderson, J. (1995), Computational Fluid Dynamics, the Basics with Applications, McGraw Hill.

Balasubramanian, R. et al (2016), Magnetohydrodynamic Flow Control of a Hypersonic Cruise Vehicle Based on AJAX Concept, ARC, Aerospace Research Central, V.5.3.4.

Balasubramanian, R. et al (2017), Mitigation of shock-induced flow separation using Magneto-hydrodynamic flow control, Sadhana, Vol. 42.3.

Chutia1, M. \& Deka, P. (2014), Numerical Solution of MHD Flow in an Insulated Rectangular Duct under the Action of Strong Transverse Magnetic Field by Finite Difference Method, International Journal of Energy \& Technology 6.10 .

Fomichev, V. \& Yadrenkin, M. (2017), Strong MHD-Interactions in Hypersonic Flows near Bodies, AIP Conference Proceedings.

Lee, L., Huerta, M. \& Zha, G. (2010), Low Rem 3-D MHD Hypersonic Equilibrium Flow Using High Order WENO Schemes, AIAA 229.

Lindsey, M. (2006), Assessing the Potential for Improved Scramjet Performance Through Application of Electromagnetic Flow Control, Air Force Institute of Technology.

Zhang, W. et al (2019), Edge-Based Finite Element Modeling of MGH-Based Propulsion Systems, AIAA ARC (Aerospace Research Central) V.57.7.

Pengyu, Y. et al (2016), Investigation of MHD power generation with supersonic non-equilibrium RF discharge, Chinese Journal of Aeronautics, Volume 29, Issue 4.

Shah, S., Hussain, S. and Sagheer, M. (2016), MHD effects and heat transfer for the UCM fluid along with Joule heating and thermal radiation using Cattaneo-Christov heat flux model, AIP Advances 6, 085103.

Shneider, M. et al (2004), Analysis of Magnetohydrodynamic Control of Scramjet Inlets, AIAA JOURNAL, Vol. 42, No. 11.

Omar Yousef Al-Thehabey: a PhD student in aerospace engineering, started in the Fall semester 2019, at the University of Cincinnati in Cincinnati, Ohio USA. Has a BS degree in mechanical engineering, a Master of Science in computer science, and graduated with a Master of Science in aerospace engineering in August 2018 from the University of Alabama in Tuscaloosa, USA. Worked as a consultant in software development on a number of projects at Halliburton, Hudson Products, Baker Hughes, Capstone Engineering, Dell, and Mayo Clinic. Interests include numerical methods, engineering analysis, software development, and software engineering. 